\title{
Propiedades psicométricas y baremación de la prueba ENFEN en zonas rurales y urbanas de Tunja (Colombia)*
}

\author{
Psychometric properties and standardization of the ENFEN \\ test in rural and urban areas of Tunja city (Colombia)
}

\section{Lucía Carlota Rodríguez Barreto \\ ORCID: 0000-0003-1306-6909}

Javier Humberto Parra Pulido** ORCID: 0000-0003-1632-2824

Julián David Chinome Torres ORCID: 0000-0003-3467-7055

Gina Paola Fonseca Estupiñán ORCID: 0000-0002-8408-3485 Universidad Pedagógica y Tecnológica de Colombia

Recibido: 12 de noviembre de 2017 Revisado: 16 de febrero de 2018 Aceptado: 24 de marzo de 2018

\section{Resumen}

El objetivo del estudio fue evaluar las propiedades psicométricas y la baremación de la prueba Evaluación Neuropsicológica de las Funciones Ejecutivas (ENFEN). Se realizó un estudio cuantitativo descriptivo de tipo psicométrico. La muestra estuvo compuesta por 367 participantes (171 niños 46,6 \%; 196 niñas $53,4 \%$ ) del área rural y urbana, con edades comprendidas entre los 6 y los 12 años $(M=8,99$ años; $D E=1,88)$. Para los resultados se utilizó el método de análisis factorial exploratorio por componentes principales para la validez de constructo y la validez de criterio con la escala de funciones ejecutivas de la prueba ENI. El análisis factorial exploratorio arrojó un solo factor en la prueba que explicó el $58,86 \%$ de la varianza. Se presentan correlaciones entre las pruebas ENI y ENFEN, entre todas las variables. 
La prueba ENFEN es una prueba válida para evaluar las funciones ejecutivas en niños del área urbana y rural la cual puede ser utilizada en el ámbito clínico e investigativo.

Palabras clave: Evaluación neuropsicológica, Baremos, Funciones Ejecutivas, área rural y urbana.

\section{Abstract}

This research aimed to evaluate the psychometric properties and standardization of the Neuropsychological Assessment of Executive Functions in Children Test (ENFEN in Spanish). This was a non-experimental quantitative and psychometric study with a convenience sample of 367 participants (171 children and 196 girls, $46,6 \%$ and 53,4 \%) of both rural and urban areas, aged 6 to 12 years $(M=8,99, S D=1,88)$. The method of exploratory factor analysis by principal components was used to establish criterion and content validity against the executive function subscale of the ENI. Exploratory factor analysis yielded a single factor explaining $58,86 \%$ of the total variance, like the original test results. Statistically significant correlations between all subscales and the ENI was found $(p<0,05)$. Results show that the ENFEN is a valid tool for assessing executive functions in children of urban and rural areas which can be used in clinical and research field test.

Keywords: Rural Areas, Standarization, Neuropsychology, Child Develompment.

\section{Introducción}

Las Funciones Ejecutivas (FE) son las encargadas de organizar y dirigir las actividades tanto cognitivas como conductuales de alto orden (Betancur, Molina \& Cañizales, 2016; Portellano \& García, 2014), abarcando procesos cognitivos que tienen que ver con la conducta consciente y planificada dirigida a metas. También se han relacionado con respuestas adecuadas en situaciones novedosas 0 la inhibición de conductas inadecuadas, las cuales requieren de un control a nivel emocional de pensamiento y conductual (Risso, García, Montserrat, Brenlla, Peralbo, \& Barca,, 2015). Desde una propuesta integradora, se definen como un sistema multimodal que permiten el logro de nuevos objetivos a partir de la resolución de problemas de forma novedosa, la programación, predicción de consecuencias y toma de decisiones (Portellano \& García, 2014; Tirapu, Climent, Luna, Bombín, Cifuentes, \& Díaz, 2014).

En el estado actual de la evaluación neuropsicológica en Colombia, la mayoría de las pruebas, si no la totalidad, son elaboradas y normalizadas en países extranjeros, lo que implica que se tengan en cuenta para su construcción características y variables de lenguaje, ocio económicas y cultu- rales de estos; dentro de las limitaciones que existen de instrumentos de evaluación neuropsicológica están las prueba que permitan evaluar las $\mathrm{FE}$ en los rangos de edad de 6 a 12 años, en niños sin patología o con algún déficit derivado de alteraciones cognitivas o emocionales, retraso madurativo por daño o disfunción cerebral en las áreas involucradas en las áreas prefrontales. Esto ha generado dificultades no solo en el diagnóstico adecuado, sino en los procesos de rehabilitación, influyendo además en la economía del sistema de salud (Arango \& Rivera, 2015; González \& Morales, 2015).

Teniendo en cuenta lo anterior y la importancia que conlleva contar con herramientas adaptadas al contexto de la población que se pretende evaluar, los instrumentos deben contar con aspectos psicométricos como la fiabilidad, validez, sensibilidad de la prueba, baremos u obtención de datos normativos en una muestra representativa (Arango \& Rivera, 2015; Aragón, 2012; Gregory, 2012).

Al respecto, la Evaluación Neuropsicológica de las Funciones Ejecutivas (ENFEN) aparece como una prueba que evalúa el constructo de FE en la edad infantil desde una visión multimodal (Portellano \& 
García, 2014), a partir de sus escalas orientadas a la evaluación atencional, memoria de trabajo, planificación, inhibición, fluidez verbal y flexibilidad mental, componentes ampliamente aceptados como características funcionales de las FE (Bausela, 2014; Betancur, Molina, \& Cañizales, 2016; Flores, Castillo \& Jiménez, 2014; Portellano, Martínez \& Zumárraga, 2009). Hasta la fecha, para la población colombiana no existen baterías normalizadas que evalúen este constructo.

A partir de lo anterior y como una forma de subsanar las dificultades en la evaluación, se presenta como alternativa la evaluación de las propiedades psicométricas y la baremación de la prueba ENFEN (Portellano, Martínez \& Zumárraga, 2009), en niños del área rural y urbana de la ciudad de Tunja (Colombia).

\section{Método}

\section{Diseño}

Se realizó un estudio descriptivo no experimental de tipo psicométrico ya que se buscó especificar las propiedades y las características de la prueba ENFEN, obteniendo a su vez, la baremación para la población Boyacense (Clark-Carter, 2002)

\section{Participantes}

La muestra estuvo conformada por 367 niños y niñas del área rural y urbana de tres instituciones educativas de la ciudad de Tunja. Dichas instituciones fueron seleccionadas por la accesibilidad a las poblaciones y debido a los tipos de sistemas educativos de carácter público y privado establecidos en Colombia (Ley 115 del Congreso de Colombia, 1994), de los cuales 140 (38,1\%) pertenecían a una institución privada, 65 (17,7 \%) pertenecían a institución pública y $162(44,1 \%)$ a una institución pública del área rural.

La distribución por sexo fue de 171 varones $(46,6 \%)$ y 196 mujeres $(53,4 \%)$, con edades comprendidas entre los 6 y los 12 años $(M=8,99$ años; $D E=1,88)$. La selección de los participantes se llevó a cabo a partir de un muestreo no aleatorio e intencional que tuvieran una edad comprendida entre los 6 y 12 años de los grados primero de primaria a séptimo bachillerato, y que cumplieran los criterios de inclusión del estudio.

Los criterios de inclusión fueron: (a) que el participante fuera estudiante de la institución seleccionada para llevar a cabo la investigación; (b) tener edades entre los 6 y 12 años durante la aplicación de las pruebas (c) tener firmado el consentimiento informado por parte de los padres y/o representantes legales y la participación voluntaria del niño/a.

Los criterios de exclusión fueron: (a) que el participante tuviera alguna limitación motriz, sensorial o cognitiva que impidiera realizar la prueba; (b) que no llevara consentimiento informado firmado por los padres y/o representante legal para participar en la investigación y/o que el niño/a no deseara participar (c) no saber leer ni conocer los números de 1 hasta 21 debido a que se requieren dichos conocimientos para la aplicación de una de las subescalas.

\section{Instrumentos}

Para el desarrollo de este estudio se empleó la Evaluación Neuropsicología de las Funciones Ejecutivas en Niños, ENFEN (Portellano, Martínez \& Zumárraga, 2009). La ENFEN es una batería compuesta por 4 pruebas que permite evaluar las FE en una población hispanohablante de niños escolares entre las edades de 6 y 12 años; es de aplicación individual y toma un tiempo de evaluación que oscila entre 20 y 30 minutos. Los resultados generales se transforman a puntuaciones típicas expresadas en decatipos. Para cada subescala, los decatipos contienen una descripción cualitativa de las puntuaciones que ayuda a interpretar los resultados; estos decatipos (distribución de 1 a 10) tienen una media de 5,5 puntos con una desviación típica de 2 . Las pruebas utilizadas incluyen: Fluidez, Senderos, Anillas e Interferencia.

La validación del instrumento español se realizó con 837 participantes, de los cuales 343 fueron hombres (41\%) y 494 mujeres (59\%), se incluyeron 
niños de diferentes estratos socioeconómicos e inmigrantes de la ciudad de Madrid, por lo que los autores consideran que puede ser una muestra representativa de los escolares españoles. La edad media de la muestra fue de 115,75 meses, DT $=20,03$ meses (9,64 años). La edad media de los niños fue de 116,63 meses DT $=20,57$ y para las niñas de 115,16 meses DT $=19,65$.

A su vez, se utilizó la batería de Evaluación Neuropsicológica Infantil- ENI (Matute, Rosselli, Ardila \& Ostrosky, 2007). Para esta investigación se utilizaron únicamente la subescalas de FE en la ENI, que está compuesta por las subescalas de fluidez, flexibilidad cognitiva, y planeación y organización.

\section{Procedimiento}

Una vez se realizó el contacto previo de autorización, durante la segunda fase se administraron las pruebas. Se tuvieron en cuenta factores medioambientales adecuados, las pruebas fueron aplicadas por dos psicólogos y un estudiante de último año de psicología.

Todos los aplicadores fueron entrenados por una profesional especializada. La administración individual de cada una de las pruebas osciló entre 30 a 45 minutos.

Durante la tercera fase se realizaron los análisis estadísticos pertinentes, y se hizo la entrega de los resultados a las instituciones educativas así como a la entidad financiadora del proyecto de investigación.

\section{Análisis de datos}

Se realizó un análisis factorial exploratorio mediante la prueba Kaiser- Meyer- Olkin y la prueba de esfericidad de Barlett se realizó un análisis de la validez de constructo. La validez de criterio se llevó a cabo mediante el estadístico rho de Spearman. Para establecer diferencias entre las distintas variables, se realizó un análisis mediante el estadístico ANOVA de $2 \times 7$, para la variable sexo vs edad y el tipo de institución (urbano y rural) vs edad. Para la obtención de baremos se utilizaron análisis de frecuencias con el fin de establecer los decatipos en cada una de las escalas.

\section{Resultados}

A partir de las correlaciones entre las distintas pruebas se llevó a cabo un análisis factorial exploratorio por el método de componentes principales. Para su realización se incluyeron las puntuaciones directas de las pruebas. Las correlaciones entre las puntuaciones de anillas y el resto de las escalas fueron negativas (ver tabla 2), debido a que la puntuación de dicha prueba se calcula con tiempo, mientras que las demás en puntuaciones correctas. Por lo tanto, se espera que, entre mejor ejecución en la escala de anillas, menor tiempo. 
Tabla 1.

Distribución de la Frecuencia según edad, área y sexo.

\begin{tabular}{|c|c|c|c|c|c|c|c|c|c|c|c|c|c|c|}
\hline \multirow[b]{3}{*}{ Edad } & \multicolumn{6}{|c|}{ Zona Urbana } & \multicolumn{6}{|c|}{ Zona Rural } & \multirow{2}{*}{\multicolumn{2}{|c|}{ Total }} \\
\hline & \multicolumn{2}{|c|}{ Hombres } & \multicolumn{2}{|c|}{ Mujeres } & \multicolumn{2}{|c|}{ Subtotal } & \multicolumn{2}{|c|}{ Hombres } & \multicolumn{2}{|c|}{ Mujeres } & \multicolumn{2}{|c|}{ Subtotal } & & \\
\hline & $\mathrm{n}$ & $\%$ & $\mathrm{n}$ & $\%$ & $n$ & $\%$ & $\mathrm{n}$ & $\%$ & $\mathrm{n}$ & $\%$ & $\mathrm{n}$ & $\%$ & $n$ & $\%$ \\
\hline 6 & 7 & 1,91 & 18 & 4,90 & 25 & 9,35 & 10 & 2,72 & 7 & 1,91 & 17 & 4,63 & 42 & 11,44 \\
\hline 7 & 16 & 4,36 & 16 & 4,36 & 32 & 19,01 & 9 & 2,45 & 12 & 3,27 & 21 & 5,72 & 53 & 14,44 \\
\hline 8 & 22 & 5,99 & 12 & 3,27 & 34 & 19,60 & 9 & 2,45 & 13 & 3,54 & 22 & 5,99 & 56 & 15,26 \\
\hline 9 & 15 & 4,09 & 13 & 3,54 & 28 & 14,48 & 16 & 4,36 & 23 & 6,27 & 39 & 10,63 & 67 & 18,26 \\
\hline 10 & 17 & 4,63 & 14 & 3,81 & 31 & 17,67 & 8 & 2,18 & 22 & 5,99 & 30 & 8,17 & 61 & 16,62 \\
\hline 11 & 14 & 3,81 & 11 & 3,00 & 25 & 11,43 & 6 & 1,63 & 9 & 2,45 & 15 & 4,09 & 40 & 10,90 \\
\hline 12 & 11 & 3,00 & 19 & 5,18 & 30 & 15,52 & 11 & 3,00 & 7 & 1,91 & 18 & 4,90 & 48 & 13,08 \\
\hline Total & 102 & 27,79 & 103 & 28,07 & 205 & 107,06 & 69 & 18,80 & 93 & 25,34 & 162 & 44,14 & 367 & 100 \\
\hline
\end{tabular}

Fuente: Elaboración Propia.

El índice de adecuación de la prueba Kaiser-Meyer-Olkin alcanzó el valor de 0,87 , siendo este valor adecuado para el análisis factorial. La prueba de esfericidad de Barlett mostró un valor estadísticamente significativo $(p<0,001)$. Con el crite- rio de Kaiser tuvo un resultado de 3,53 siendo el autovalor mayor que 1 , donde se extrajo un único factor, lo que da cuenta de la unidimensionalidad de las funciones ejecutivas. Este factor explica el $58,86 \%$ de la varianza total.

Tabla 2.

Correlaciones entre las puntuaciones de las pruebas de la ENFEN.

\begin{tabular}{|c|c|c|c|c|c|c|}
\hline & $\begin{array}{c}\text { Fluidez } \\
\text { Fonológica }\end{array}$ & $\begin{array}{c}\text { Fluidez } \\
\text { Semántica }\end{array}$ & $\begin{array}{c}\text { Senderos } \\
\text { Grises }\end{array}$ & $\begin{array}{l}\text { Senderos } \\
\text { Color }\end{array}$ & Anillas & Interferencia \\
\hline \multicolumn{7}{|l|}{ Fluidez Fonológica } \\
\hline Fluidez Semántica & $0,56\left(^{* *}\right)$ & & & & & \\
\hline Senderos Grises & $0,49\left(^{* *}\right)$ & $0,55\left(^{* *}\right)$ & & & & \\
\hline Senderos Color & $0,52\left({ }^{* *}\right)$ & $0,56\left(^{* *}\right)$ & $0,72\left(^{* *}\right)$ & & & \\
\hline Anillas & $-0,46\left({ }^{* *}\right)$ & $-0,45\left({ }^{* *}\right)$ & $-0,49\left({ }^{* *}\right)$ & $-0,56\left({ }^{* *}\right)$ & & \\
\hline Interferencia & $0,41\left({ }^{* *}\right)$ & $0,39\left(^{* *}\right)$ & $0,42\left({ }^{* *}\right)$ & $0,52\left(^{* *}\right)$ &,- 368 & \\
\hline
\end{tabular}

Fuente: Elaboración propia. 
La validez de criterio fue llevada a cabo con la subprueba de funciones ejecutivas de la ENI. Los resultados obtenidos por medio de la prueba de Pearson muestran correlaciones estadísticamente significativas $(p<0,05)$ entre todas las variables (véase tabla 3). A su vez, dichas correlaciones oscilan entre fuertes y débiles. La escala de anillas de la ENFEN, los números de ensayos, número de errores y perseveraciones pertenecientes a la subescala de flexibilidad cognitiva de la ENI obtuvieron correlaciones negativas ya que los resultados de velocidad de procesamiento y tiempo de ejecución, tienden a disminuir conforme aumenta la edad.

Tabla 3.

Matriz factorial de las pruebas ENFEN

\begin{tabular}{lcc}
\hline \multicolumn{1}{c}{ Prueba } & Componente & Comunalidad \\
\hline Fluidez Fonológica & 0,75 & 0,56 \\
\hline Fluidez Semántica & 0,77 & 0,59 \\
\hline Anillas & 0,72 & 0,53 \\
\hline Sendero Gris & 0,81 & 0,66 \\
\hline Sendero Color & 0,86 & 0,74 \\
\hline Interferencia & 0,66 & 0,44 \\
\hline
\end{tabular}

Fuente: Elaboración Propia.

Los resultados de la comparación en la ejecución de la prueba entre el tipo de institución ponen de manifiesto las diferencias estadísticamente significativas entre el grupo urbano y rural en todas las subescalas de la ENFEN, con mejores resultados en la muestra urbana en la totalidad de las escalas. A su vez, en la variable de edad se evidencian diferencias estadísticamente significativas entre los 6 y los 12 años. En la variable sexo, solo se encuentran diferencias estadísticamente significativas en la escala de anillas (véase tabla 5).

Los efectos de interacción entre la edad y el sexo únicamente se presentaron en la fluidez fonológica $\left[F_{(6,353)}=2,64 ; p=0,01\right]$, indicando que la diferencia en la ejecución en esta escala entre los sexos no parecen ser la misma a lo largo de todas las edades. No se evidenciaron efectos de interacción entre el sexo y la edad en las escalas de fluidez semántica $\left[F_{(6,353)}=1,12 ; p=0,35\right]$, sen- deros grises $\left[F_{(6,353)}=0,53 ; p=0,79\right]$, senderos a color $\left[F_{(6,353)}=0,89 ; p=0,50\right]$, anillas $\left[F_{(6,353)}=0,52\right.$; $p=0,79]$ e interferencia $\left[F_{(6,35)}=1,09 ; p=0,36\right]$.

Los resultados permiten utilizar la edad y la escolaridad como criterio diferenciador para la construcción de los baremos. Los análisis de contraste a posteriori fueron llevados a cabo con la prueba de Bonferroni para las escalas que cumplieron el supuesto de homocedasticidad de las varianzas (fluidez semántica e interferencia) y con el estadístico Games-Howell para los que no cumplieron dicho criterio obtenido por medio de la prueba de Levene (fluidez fonológica, senderos grises, senderos a color y anillas).

Las pruebas post hoc con el contraste de Bonferroni en la fluidez semántica muestran diferencias entre los grupos de edad Específicamente, la edad de 6 años no presenta diferencia con el grupo de 7 años (dif $=-1,06 ; p=1$ ) pero se evidencian diferencias estadísticamente significativas con las demás edades (dif $_{6-8 \text { años }}=-3,15 ;$ dif $_{6-9 \text { años }}=-3,31$; $\operatorname{dif}_{6-10 \text { años }}=-4,24 ;$ dif $_{6-11 \text { años }}=-7,33 ;$ dif $_{6-12 \text { años }}=-6,87$; $p<0,001)$. El grupo de 7 años no presentó diferencias con el grupo de 8 años (dif $=-2,09$; $p=0,56)$ pero mostró diferencias significativas con los grupos de edad superiores (dif7-9 años = $-2,25 ;$ dif $_{7-10 \text { años }}=-3,18 ;$ dif $_{7-11 \text { años }}=-6,26 ;$ dif $_{7-12 \text { años }}$ $=-5,81 p<0,05)$. No se encontraron diferencias entre los grupos de 8,9 y 10 años $d i f_{8-9 \text { años }}=-0,15$; $d i f_{8-10 \text { años }}=-1,09 ; \operatorname{dif}_{9-10 \text { años }}=-0,93 p>0,05$ ) pero se evidencia diferencias con los restantes grupos de edad. No se identificaron diferencias entre las edades de 11 y 12 años (dif $=0,45 ; p=1.0$ ).

La escala de interferencia presentó diferencias entre todos los grupos de edad. Concretamente a la edad de 6 años no existieron diferencias con las edades de 7 y 8 años $\left(\operatorname{dif}_{6-7 \text { años }}=-8,65 p=0,44\right.$; $\operatorname{dif}_{6-8 \text { años }}=-7,65 p=0,82$ ), a su vez, el grupo de 7 y 8 años no presentó diferencias con las edades de 9 (dif $_{7-9 \text { años }}=-2,45 p=1,00 ;$ dif $_{8-9 \text { años }}=-3,45$ $p=1,00)$ y 10 años $\left(d i f_{7-10 \text { años }}=-9,29 p=0,13 ; \operatorname{dif}_{8 \text {. }}\right.$ 10 años $=-10,30 p=0,05)$. El grupo con edad de 10 años, no presentó diferencias con las medidas del grupo de 11 años (dif $=-9,67 ; p=1,00$ ) y este a su vez, no difirió con el grupo de 12 años $(p=1,00)$ (dif $=-3,54 ; p=1,00)$. 
Tabla 4.

Validez Convergente ENFEN y ENI

\begin{tabular}{lcccccc}
\hline & $\begin{array}{c}\text { Fluidez } \\
\text { Fonológica }\end{array}$ & $\begin{array}{c}\text { Fluidez } \\
\text { Semántica }\end{array}$ & $\begin{array}{c}\text { Senderos } \\
\text { grises }\end{array}$ & $\begin{array}{c}\text { Senderos } \\
\text { color }\end{array}$ & Anillas & Interferencia \\
\hline Fluidez grafica semántica & $0,46^{* *}$ & $0,52^{* *}$ & $0,50^{* *}$ & $0,54^{* *}$ & $-0,433^{* *}$ & $0,32^{* *}$ \\
\hline Fluidez gráfica no semántica & $0,43^{* *}$ & $0,43^{* *}$ & $0,47^{* *}$ & $0,49^{* *}$ & $-0,428^{* *}$ & $0,37^{* *}$ \\
\hline Flexibilidad Cognitiva Ensayos & $-0,113^{*}$ & $-0,116^{*}$ & $-0,145^{* *}$ & $-0,151^{* *}$ & 0,02 & $-0,124^{*}$ \\
\hline Flexibilidad Cognitiva Errores & $-0,248^{* *}$ & $-0,320^{* *}$ & $-0,332^{* *}$ & $-0,337^{* *}$ & $0,29^{* *}$ & $-0,207^{* *}$ \\
\hline $\begin{array}{l}\text { Flexibilidad Cognitiva } \\
\text { Categorías }\end{array}$ & $0,23^{* *}$ & $0,36^{* *}$ & $0,28^{* *}$ & $0,32^{* *}$ & $-0,310^{* *}$ & $0,19^{* *}$ \\
\hline $\begin{array}{l}\text { Planeación Respuestas Co- } \\
\text { rrectas }\end{array}$ & $0,25^{* *}$ & $0,35^{* *}$ & $0,34^{* *}$ & $0,34^{* *}$ & $-0,388^{* *}$ & $0,19^{* *}$ \\
\hline $\begin{array}{l}\text { Planeación Perseveraciones } \\
\text { Planeación Diseños Correctos }\end{array}$ & $-0,282^{* *}$ & $-0,382^{* *}$ & $-0,408^{* *}$ & $-0,419^{* *}$ & $0,33^{* *}$ & $-0,205^{* *}$ \\
\hline Nota: & $0,26^{* *}$ & $0,28^{* *}$ & $0,29^{* *}$ & $-0,180^{* *}$ & $0.15^{* *}$ \\
\hline
\end{tabular}

Nota: ${ }^{* *}$ Correlación significativa al nivel $p<0,01$ (bilateral).

Fuente: Elaboración propia.

Los contrastes a posteriori de Games-Howell pusieron de relieve diferencias entre los grupos de edad en la subescala de fluidez fonológica. No se encontraron diferencias entre la edad de 6 y 7 años (dif $=-0,52 ; p=0,84$ ). Los grupos de 8,9 y 10 años presentaron un desempeño similar en la media de palabras reproducidas $\left(\right.$ dif $_{8-9 \text { años }}=-0,24$; $p=0,99$ y $\left.\operatorname{dif}_{8-10 \text { años }}=-1,3 ; p=0,25\right)$. Mientras que los grupos de 10, 11 y 12 años, no presentaron diferencias estadísticamente significativas $\left(\right.$ dif $_{10-11}$ años $=-0,87 p=0,83 ; \operatorname{dif}_{10-12 \text { años }}=-1,65 p=0,18 ;$ dif $_{11-12}$ años $=-0,77 p=0,94)$.

En la subescala de senderos grises, también se presentaron diferencias entre el tiempo de ejecución en las diferentes edades, de la siguiente manera: la edad de 6 y 7 años no mostraron diferencias estadísticamente significativas (dif $=$ $-2,49 ; p=0,68$ ), a su vez la edad de 7 años tampoco presentó diferencias con el grupo de edad de 8 años (dif $=-4,67 ; p=0,53$ ). El grupo de 8 años tuvo desempeños similares en la ejecución de esta escala con los grupos de edad de 9 y 10 años (dif $=-0,76 ; p=0,99$ y dif $=-3,70 ; p=0,19$ ). Además no existen diferencias entre los grupos de 10,11 y $12\left(d i f_{10-11 \text { años }}=-1,17 p=0,98 ; d i f_{10-12 \text { años }}=\right.$ $-4,38 p=0,18 ; d_{1 i f}{ }_{11-12 \text { años }}=-3,21 p=0,39$ )
Al igual que las anteriores escalas, senderos a color evidencia diferencias entre todas las edades. A los 6 años, la media de ejecución en esta escala fue similar al grupo de 7 años (dif $=-1,64$; $p=0,22$ ). Así, no se presentaron diferencias estadísticamente significativas entre los grupos de 8 y 9 años (dif $=-1,63 ; p=0,31$ ), tampoco en la edad de 9 y 10 años (dif $=-1,64 ; p=0,46$ ). A su vez, el grupo de edad de 10 años tampoco presentó diferencias en la media de ejecución con la edad de 11 y 12 años (dif ${ }_{10-11 \text { años }}=-3,07 \mathrm{p}=0,07 ;$ dif $_{10-12}$ $=-2,58 p=0,09)$ y los grupos de 11 y 12 años emplean tiempos similares en la ejecución de la escala $(p=1,00)$.

En las subescala de anillas, los contrastes a posteriori se observaron diferencias entre los grupos de edad, los niños de 6 y 7 años mostraron diferencias significativas entre el tiempo de ejecución con las edades restantes $\operatorname{dif}_{6-7 \text { años }}=79,48 ;$ dif $_{6-8}$ años $=119,78 ;$ dif $_{6-9 \text { años }}=119,47 ;$ dif $_{6-10 \text { años }}=153,17$; $\operatorname{dif}_{6-11 \text { años }}=161,39 ;$ dif $_{6-12 \text { años }}=167,91 ; p<0,05$ ), en los 8 años existió una media de ejecución similar con los de 9 (dif $=-0,31 ; p=1,0)$ y 10 años (dif $=$ $33,39 ; p=0,05)$. A su vez, en el grupo de 10 años, no se encontraron diferencias estadísticamente significativas en los de 11 (dif $=8,21 ; p=0,95$ ) y 12 
años (dif $=14,74 ; p=0,52$ ) teniendo estos últimos grupos de edad, desempeños similares ( $d i f=6,52$; $p=0,99$ ).

Los baremos fueron obtenidos agrupando los grupos de edad y el tipo de zona. Dentro de los resultados obtenidos en las diferentes subescalas, no se evidenciaron diferencias entre las edades de 11 y 12 años en ninguna de las pruebas de la ENFEN, por lo que se unificaron los baremos para estos dos grupos de edad. Los baremos para cada grupo de edad y zona se pueden ver de las tablas 5 a la 10.

\section{Discusión}

El objetivo de la investigación fue analizar las propiedades psicométricas de la batería ENFEN, y por otra parte, diseñar en donde fuera necesario los baremos en una muestra de escolares de la ciudad de Tunja (Boyacá). El uso de éstos datos podría contribuir a la reducción de sesgos interpretativos, asociados a las diferencias socio-culturales, existentes en la población donde se realizó la prueba original, y las particularidades contextuales de la población en donde se requiera su uso, esto permitirá tener un punto de referencia con un grupo representativo con características similares, (Ardila \& Ostrosky, 2012; Cohen \& Swerdlik, 2001; Hebbn \& Milberg, 2011; Hernández, Fernández \& Baptista, 2010; Portellano \& García, 2014).

Previo al análisis factorial, se desarrolló una matriz de correlaciones entre las subescalas del instrumento, donde se encontró que existen correlaciones entre todas las sub escalas de manera positiva exceptuando la sub escala de anillas con las demás, esto puede ser explicado, porque la variable edad es inversa al tiempo de ejecución, dichas correlaciones superan el valor de 0,3 lo que sugiere un acertado uso del análisis factorial exploratorio (Mendéz \& Rondón, 2012; Pérez \& Medrano, 2010).

A partir de esta correlación se ejecutó el índice de adecuación Kaiser-Meyer-Olkin, (KMO) el cual presentó un valor de 0,87 un valor interpretado como meritorio, (Mendéz y Rondon, 2012) lo que supone, según Hair, Anderson, Tatham y Black,
(1999) una intercorrelación satisfactoria y adecuada; asociado a esto, la significancia de la prueba de Barlett fue de $p<0,00$, lo que indica que las variables están lo suficientemente inter-correlacionadas para hacer uso del análisis factorial exploratorio (Everitt y Wikes, 2001), el criterio Kaiser indica un valor de 3,53, lo que indicaría una unidimensionalidad del constructo, en este caso, Funciones Ejecutivas, tal como lo encontró en la prueba original (Portellano, Martínez \& Zumárraga, 2009) el factor hallado explica el 58,86 \% de la varianza total.

La validez de criterio se realizó con la subescala de funciones ejecutivas de la ENI (Matute et al.,2007), se evidenciaron correlaciones interpretadas como fuertes y débiles, con un nivel de significación de $p<0.05$ entre todas las variables (Hernández, Fernández, \& Baptista, 2010), se observaron correlaciones negativas en la subescala de anillas de la ENFEN. En la prueba ENI se observaron coeficientes de correlación negativos en las subescalas pertenecientes a flexibilidad cognitiva, es el caso de: números de ensayos, número de errores y perseveraciones, existiendo una posible relación inversa entre la velocidad de procesamiento y tiempos de ejecución de las pruebas con la edad, pues se estima que a medida que incrementa la edad el procesamiento es mayor.

Los resultados ponen de manifiesto la necesidad de tener un criterio diferenciador para la obtención de los baremos en las variables edad y tipo de zona, en este caso los participantes del área urbana y los escolares del área rural.

La edad, según describe Semrud-Clikeman y Teeter-Allison, (2011) es un criterio de diferenciador en las funciones ejecutivas, y constituye un criterio de validez de convergencia y de divergencia, puesto que a una edad específica se esperaría que el menor ya tenga ciertas habilidades (convergencia) y no pueda realizar otras, (divergencia) pero se esperaría que las adquiera a medida que avanza su edad, de igual manera que observó Portellano, Martínez y Zumárraga (2009) en la prueba original, no existen diferencias en el desempeño entre los niños de 11 y 12 años, por lo que se realiza un mismo baremo para estas edades. 
Para identificar las posibles diferencias existentes entre los escolares del área urbana y rural, se realizó un análisis de comparación de medias a través del estadístico t-student, el desempeño entre las dos muestras difiere significativamente en todas las subescalas de la ENFEN y evidencian un mejor desempeño en los participantes escolarizados en la zona urbana, por tal motivo, se diseñaron los baremos para cada una de las zonas estudiadas.

Estudios describen diferencias en el desempeño neuropsicológico entre estas dos zonas, favoreciendo el desempeño neuropsicológico de los niños que residen en las zonas urbanas (Galindo, Solovieva, Machinskaya \& Quintanar, 2013; Matalinares, et al., 2007; Soto, 2011), explicado a partir de las posibles diferencias socio-económicas y culturales, que pueden generar características contextuales particulares que intervienen en el neurodesarrollo (Huanca, 2012; Morante \& Soto, 2013; Quintanar, Lázaro \& Solovieva 2002; Quintanar, Solovieva \& Lázaro 2008; Sanhueza, 2006), lo que implica tener precaución en el uso de los test psicométricos y su interpretación, por lo que también se recomienda realizar baremos para cada zona en particular (Martín \& Oros, 2013).

En el presente estudio los estadísticos evidencian la necesidad de realizar baremos para cada una de las zonas estudiadas debido a las diferencias halladas y que concuerdan con las teorías referenciadas. Esta baremación permitirá contar con herramientas a los profesionales en neuropsicología en la práctica clínica o investigativa que hagan uso de la prueba ENFEN, se espera que el uso de estos baremos contribuyan en diferentes procesos, como el abordaje terapéutico, diseños de intervención, rehabilitación y seguimiento. Sin embargo, es necesario aclarar que la interpretación del desempeño, seguirá sujeta al criterio del profesional que haga uso de ella, igualmente se recomienda tener las precauciones necesarias para dar una aproximación diagnóstica, como es común en la práctica psicológica y con cualquier tipo de test psicométrico.

En conclusión, las FE son un constructo que reúne las habilidades cognoscitivas que permiten anticipar, iniciar, retroalimentar el comportamiento y tener flexibilidad cognoscitiva (Acosta, Avendaño, Martínez \& Romero, 2014), se caracterizan por ser funciones complejas y de alto orden, (VerdejoGarcía \& Bechara, 2010; Filippetti \& López, 2013) por tal motivo, se ve la necesidad de tener una prueba psicométrica baremada para dar una aproximación a un diagnóstico, y que como todos los demás constructos, existe la obligación profesional de realizar una valoración objetiva y científica, ya que demanda el adecuado uso de los test psicométricos (Ardila \& Ostrosky, 2012).

Se recomienda seguir realizando estudios de baremación de diferentes escalas psicométricas, ya que se evidencian cambios a nivel socio-cultural que pueden sesgar la aplicación e interpretación del desempeño de quien se le aplica.

A pesar de que la presente baremación es una herramienta útil para la evaluación de las $\mathrm{FE}$, es necesario que los profesionales complementen la información con diferentes componentes neuropsicológicos, psicológicos y psicométricos, para tener un diagnóstico más acertado (Arango et al., 2014). 


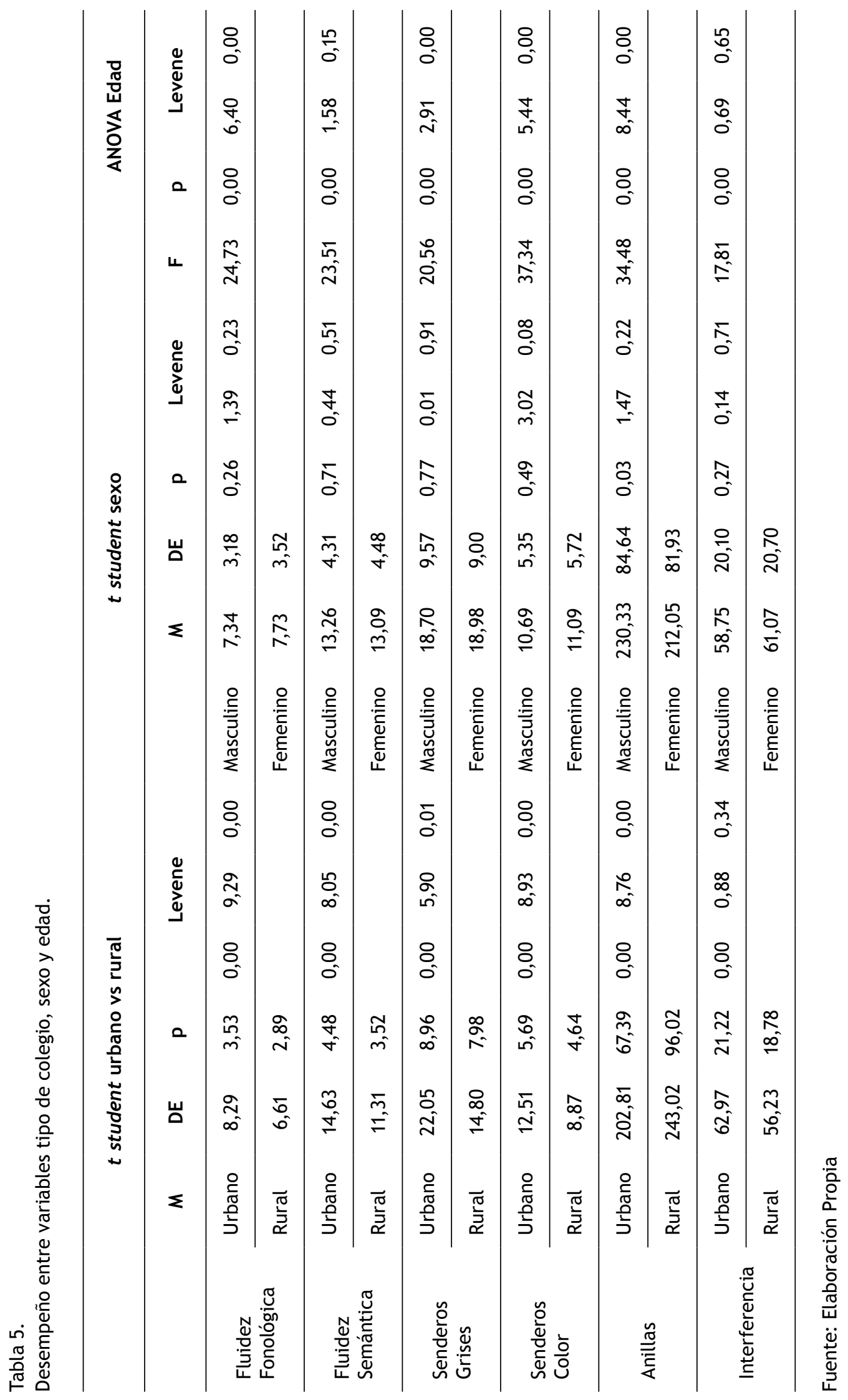




\section{Referencias}

Acosta, M. R., Avendaño, B. L., Martínez, M., \& Romero, L. M. (2014). Análisis psicométrico del test de "fluidez de diseños de Ruff" en población universitaria de Bogotá. Acta Colombiana de Psicología, 17(1), 45-52. doi: 10.14718/ACP.2014.17.1.5.

Arango, J., Rivera, D., Panyavin, I., Rogers, H., Utria,O., ... \& Perrin, P. (2014). A National Survey of the Practice of Neuropsychology in Colombia. Archives of Clinical Neuropsychology, 29(6), 534-534. Doi: http://dx.doi. org/10.1093/arclin/acu038.36

Arango, J. \& Rivera, D. (2015). Neuropsicología en Colombia: Datos normativos, estado actual y restos a futuro. Colombia: Universidad de Manizales.

Aragón, L. (2012). Evaluación psicológica. historia, fundamentos teóricos, conceptuales y Psicometría. México D.F.: Manual Moderno.

Ardila, A., \& Ostrosky, F. (2012). Guía para el diagnóstico neuropsicológico. Miami, Florida - México DF: Florida International University - Universidad Nacional Autónoma de México.

Bausela, E. (2014). Funciones ejecutivas: nociones del desarrollo desde una perspectiva neuropsicológica. Acción psicológica, 11(1), 21-34. http://dx.doi.org/10.5944/ap.1.1.13789

Betancur, M., Molina, D., \& Cañizales, L. (2016). Entrenamiento cognitivo de las funciones ejecutivas en la edad escolar. Revista Latinoamericana de Ciencias Sociales, Niñez y Juventud, 14(1), 359-368. doi:10.11600/16927 15x.14124160615

Clark-Carter, D. (2002). Investigación Cuantitativa en Psicología: Del diseño experimental al reporte de investigación. México: Oxford University Press.

Cohen, R., \& Swerdlik, M. (2001). Pruebas y evaluacion psicologicas. España: McGraw- Hill.
Congreso de Colombia. (1994). Ley 115 de 1994. Por la cual se expide la ley general de educación. Recuperado de http://www.oei.es/ quipu/colombia/Ley_115_1994.pdf

Everitt, B. S. \& Wykes, T. (2001). Diccionario de Estadística para Psicólogos. España: Ariel.

Filippetti, V. A., \& López, M. B. (2013). Las funciones ejecutivas en la clínica neuropsicológica infantil. Psicología desde el Caribe, 30(2), 380-415.

Flores, J., Castillo, R., \& Jiménez, N. (2014). Desarrollo de Funciones ejecutivas de la niñez a la juventud. Anales de Psicología, 30(2), 463-473. http://dx.doi.org/10.6018/analesps. 30.2.155471

Galindo, G., Solovieva, Y., Machinskaya, R. B \& Quintanar, L. (2013) Vusial-spatial processing in urban and rural elementary school children. International Journal of Hispanic Psychology, 6(2) 129-142.

González, Y. \& Morales, L. (2015). Estimulación cognoscitiva en las funciones ejecutivas en niños con edad comprendida entre los $6 \mathrm{y}$ los 12 años de edad (Trabajo de especialización en Neuropsicología escolar). Institución Universitaria Politécnico Grancolombiano, Colombia.

Gregory, R. (2012). Evaluación psicológica. Historia, principios y aplicaciones. México D.F.: Manual Moderno.

Hair, J. F., Anderson, R. E., Tatham, R. L., \& Black, W. (1999). Análisis Multivariante. Madrid: Prentice Hall.

Hebben, N., \& Milberg, W. (2011). Fundamentos para la Evaluación Neuropsicológica. SonoraMéxico: Manual Moderno

Hernández, R., Fernández, C., \& Baptista, P. (2010). Metodología de la Investigación (10 . ed.). México: Editorial McGraw- Hill Interamericana. 
Huanca, D., (2012). Manual de neuropediatría GPC Basada en la evidencia. Editorial IIDENUT Lima: Perú.

Matute, E., Rosselli, M., Ardila, A., \& Ostrosky,F. (2007). Evaluación neuropsicológica infantil. México: Universidad de Guadalajara.

Martín, L. \& Oros, M. (2013). Una Experiencia de Evaluación Psicológica en una Zona Rural. Revista Latinoamericana de Ciencia Psicológica, 5(2), 150-158 doi: 10.5872/psiencia/5.2.77.

Matalinares, M., Ch, A., Arenas, C., Acosta, G., Zamora, J., ... \& Suárez, J. (2007). Lenguaje comprensivo y memoria inmediata en estudiantes de 5to y 6to grado de primaria de zona rural y urbana de Lima. Revista de investigación en psicología, 10(2), 71-83

Mendéz, M. \& Rondón, S. (2012). Introducción al análisis factorial exploratorio. Revista Colombiana de Psiquiatría, 41(1), 197-207 http://doi. org/10.1016/S0034-7450(14)60077-9

Morante, P. \& Soto, M. (2013). Discrepancias En El Rendimiento Neuropsicológico En Niños de Zona Rural y Urbana. Revista de psicología de Arequipa, 3(2) 177-182.

Pérez, E. R., \& Medrano, L. (2010). Análisis factorial exploratorio: Bases conceptuales y metodológicas. Revista Argentina de Ciencias Del Comportamiento, 2(1889), 58-66

Portellano, J. \& García, J. (2014). Neuropsicología de la atención, las funciones ejecutivas y la memoria. Madrid, España: Editorial síntesis.

Portellano, J., Martínez, R. \& Zumárraga, L (2009). Evaluación Neuropsicológica de las Funciones Ejecutivas en Niños. Madrid, España: TEA Ediciones.
Quintanar, L., Lázaro, E. \& Solovieva, Y. (2002). Evaluación neuropsicológica de escolares rurales y urbanos desde la aproximación a Luria. Revista española de neuropsicología, 4(2), 217-235.

Quintanar, L., Solovieva, Y., \& Lázaro, E. (2008). Evaluación neuropsicológica infantil breve para población hispano-parlante. Acta Neurológica Colombiana, 24(2), 31-44

Risso, A., García, M., Montserrat, D., Brenlla, J., Peralbo, M., \& Barca, A. (2015). Relaciones entre funciones ejecutivas, lenguaje y habilidades matemáticas. Revista de estudios $e$ investigación, Extracto (9), 72-78. Doi: 10.17979/ reipe.2015.0.09.577.

Sanhueza, A. (2006). Psychomotor development, environmental stimulation, and socioeconomic level of preschoolers in Temuco, Chile. Pediatric Physical Therapy, 18(2), 141-147

Semrud-Clikeman, M. \&Teeter-Allison, P. (2011). Neuropsicología infantile. Evaluación de intervención de lostrastornos neuroevolutivos. (2. Ed.). Madrid, España: Pearson Ediciones.

Soto, J. (2011). Variación consonántica en el habla urbana y rural de Ñuble. Revista de Lingüística Teórica y Aplicada 45(2): 143-165.

Tirapu, J., Climent, G., Luna, P., Bombín, I., Cifuentes, A., \& Díaz, U. (2014). Evaluación Neuropsicológica de las funciones ejecutivas mediante realidad virtual. Revista de Neurología, 5(6), 465-475.

Verdejo-García A, Bechara A. (2010) Neuropsicología de las funciones ejecutivas. Psicothema, (22) 227-35. 